\title{
AORTIC DISSECTION IN AN SLE PATIENT: MORE THAN ONE COLLAGEN DISORDER - CASE REPORT
}

Afonso Guilherme Schmidt ${ }^{1, \star}$, André Lucas Ribeiro ${ }^{1}$, Andrea Worm Furtado ${ }^{1}$, Augusto Emilio Hinterholz ${ }^{1}$, Larissa Martinelli Dullius ${ }^{1}$, Larissa Vargas Cruz $^{1}$, Claiton Viegas Brenol ${ }^{1}$, Natalia Sarzi Sartori ${ }^{1}$, Penelope Esther Palominos ${ }^{1}$

1.Hospital de Clínicas de Porto Alegre, Porto Alegre (RS), Brazil.

*Corresponding author: afonsogschmidt@gmail.com

\section{BACKGROUND}

Vascular Ehlers-Danlos syndrome (VEDS) is caused by mutation in procollagen type 3 gene, namely COL3A1, generating vascular, intestinal or uterine fragility, with consequent vascular dissection, gastrointestinal perforation, or uterine rupture during pregnancy. Other features include thin skin, acrogeria, hypermobility of small joints, chronic joint dislocation, and clubfoot. It represents $4 \%$ of the Ehlers-Danlos syndrome cases. We report the case of a woman with systemic lupus erythematosus (SLE) and vEDS.

\section{CASE REPORT}

A 37-year-old woman, with lupus since 29 , characterized by thrombocytopenia, and joint and cutaneous manifestations, with recent increase in tocilizumab from 4 to $8 \mathrm{mg} / \mathrm{kg}$ because of wrist arthritis, came to the emergency department because of acute chest pain. Computed tomography angiography showed descendent aortic dissection from subclavian to iliac arteries, and aortic roots (Figure 1, arrowhead), without signs of vessel wall inflammation, such as thickening of the wall or vascular occlusion. She was off steroids, complements and platelets were normal, without signs of clinical lupus activity. She had ascendent and abdominal aortic ectasia diagnosed incidentally in previous imaging. Also, the patient was able to perform dorsiflexion of the 5 th fingers by more than $90^{\circ}$, touch her forearm with her thumb, and hyperextend her knees by more than $10^{\circ}$. She was seen by Genetics and the diagnosis of EDS was established. Endovascular repair was performed successfully because of refractory arterial hypertension, and the patient was discharged well and without the need for increased immunosuppression.

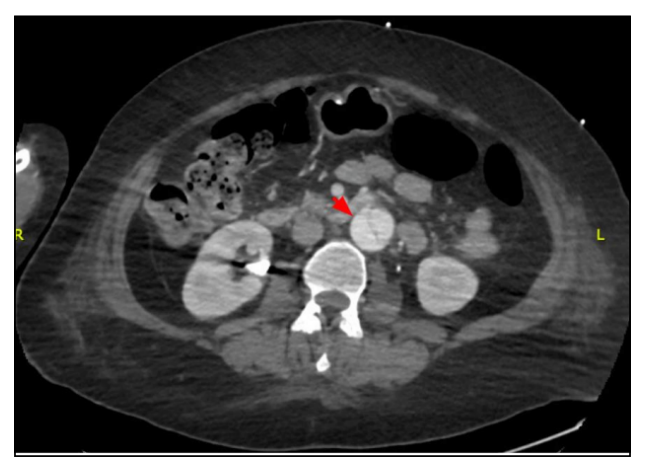

Figure 1. Computed tomography angiography.

\section{CONCLUSION}

There are just a few related cases of the association of these diseases in the literature, being one autoimmune and the other a genetic disorder. The differential diagnosis between these two entities is extremely important in cases of aortic dissection, and difficult when the diagnosis of SLE is already done. The therapeutic approach is also distinct for these two entities. The need for immunosuppressants may be challenging because of the risk of infection of an endovascular stent, as well as the blood pressure may be difficult to be strictly controlled if corticosteroid is needed. Our patient had a good recovery after endosurgery, but the general median lifespan is 48 years, which may be even less depending on the SLE activity. There is no robust literature data regarding the prognosis of this rare association.

\section{KEYWORDS}

Ehlers-Danlos, Lupus, Dissection.

Realização: 